\title{
Predictors of high intakes of protein-rich foods by older adults: Liking, perceived convenience and perceived value for money
}

\author{
K.M. Appleton \\ Bournemouth University, Poole House, Fern Barrow, Poole, Dorset, BH12 5BB, UK
}

Protein-specific under-nutrition is considered to affect $10-20 \%$ of UK older adults ${ }^{(1)}$, with potential detriments to health and wellbeing ${ }^{(1,2)}$. This under-nutrition is considered to result, at least in part, from low protein intakes, or low intakes of protein-rich foods ${ }^{(1,3)}$. Older adults can provide various reasons for both the high and low consumption of protein-rich foods ${ }^{(4)}$; reasons related to the foods themselves, to the individual, and to their living situation. The relative importance of each these reasons, however, remains unknown. This work investigated the importance of reasons for the consumption of four categories of protein-rich foods in relation to protein intakes, in a sample of UK older adults.

Questionnaires assessed: usual frequency of consumption of meat (red meat, white meat, processed meat), fish (white fish, oily fish, seafood), eggs, and dairy products (milk, yoghurts/ custards, hard cheeses, soft cheeses); agreement or disagreement with 30 statements assessing 15 previously identified reasons for protein consumption/non-consumption in this population ${ }^{(4)}$; and various demographic and lifestyle characteristics of relevance to food intake. One thousand questionnaires were administered by post, with three repeat postings to non-responders.

A total of 348 returned questionnaires were suitable for analysis. Respondents (149 male, 199 female, aged 65-94 years from across the UK), were representative of the UK older population at the 2011 census, in terms of gender and age. Meat was consumed 0-3 times/day (mean $=0 \cdot 6)$. Higher intakes were associated with higher liking $(\beta=0 \cdot 15, p=0 \cdot 03)$, greater perceptions of meat as convenient $(\beta=0.13, p=0.03)$, and greater perceived ability to afford meat $((\beta=0.12, p=0.04)$. Fish was consumed from 0 -twice/day (mean $=0 \cdot 3)$. Higher intakes were associated with higher liking $(\beta=0 \cdot 17, p=0 \cdot 02)$, a greater importance of freshness $(\beta=0 \cdot 16, p=0 \cdot 03)$, and a lower perception of the effort required to prepare and cook fish $(\beta=-0 \cdot 13, p=0.05)$. Eggs were consumed from 0 -once/day (mean $=$ $0 \cdot 3)$. Higher intakes were associated with greater liking $(\beta=0 \cdot 17, p=0 \cdot 03)$, perceptions of eggs as convenient $(\beta=0 \cdot 19$, p $<0 \cdot 01)$, and lower perceptions of likely spoilage and waste $(\beta=-0 \cdot 20, \mathrm{p}<0 \cdot 01)$. Dairy products were consumed from $0-3 \cdot 6$ times/day $(\mathrm{mean}=$ $1.7)$. Higher intakes were associated with higher liking $(\beta=0.26, p<0.01)$.

These findings demonstrate the importance of liking for the consumption of all protein-rich foods investigated, but also suggest high importance for convenience or effort required, and affordability or value for money. Meat, fish and eggs were more frequently consumed by those who perceived them as convenient or of little effort to prepare and cook, and meat and eggs were more frequently consumed by those who felt able to afford them or that they would likely not be wasted. Strategies to increase intakes of protein-rich foods that focus on these particular reasons, thus may demonstrate increased success. Other reasons investigated were not important.

1. Cowan DT, Roberts JD, Fitzpatrick JM, While AE, Baldwin J. (2004) Nurtitional status of older people in long term care settings: Current status and future directions. Int J Nursing Stud 41, 225-37.

2. Wolfe RR. (2012) The role of dietary protein in optimizing muscle mass, function and health outcomes in older individuals. Brit J Nutr 108, S88-93.

3. Leslie WS. (2011) Nutrition management in special populations: Improving the dietary intake of frail older people. Proc Nut Soc 70, $263-7$.

4. Best RL, Appleton KM. (2013) Investigating protein consumption in older adults: A focus group study. J Nutr Educ Behav 45, 751-5. 